\title{
Fotografia Científica Ambiental: uma perspectiva biológica
}

\section{Yuri Fanchini Messas}

Departamento de Biologia Animal, Instituto de Biologia, Universidade de Campinas, 13083-970, Campinas, São Paulo, Brasil

Correspondencia: yurimessas@gmail.com

\section{INTRODUÇÃO}

O acúmulo gradual de conhecimentos sobre princípios químicos e físicos permitiu ao ser humano utilizar a luz como matéria-prima para realizar a transposição de sua identidade visual em superfícies fotossensíveis. Esta técnica, instintivamente conhecida como Fotografia, foi recebida com muito entusiasmo pela comunidade científica desde a sua invenção no início do século XIX. Biólogos pesquisadores, particularmente, encontraram na fotografia uma inesgotável fonte de inspiração para realizar estudos objetivando a compreensão de fenômenos que ocorrem em ambientes naturais.
A fotografia e a biologia possuem historicamente diversos pontos de convergência, coevoluindo em uma interação positiva e mutualística. A Inglaterra oitocentista foi cenário de duas importantes quebras de paradigma que mudaram drasticamente a forma como o ser humano percebe e interpreta o ambiente ao seu redor (Prodger, 2009). Poucos anos após o pesquisador britânico William Henry Fox Talbot dar um grande salto evolutivo para o descobrimento do que hoje denominamos Fotografia (The pencil of Nature, Talbot, 1844), Charles Darwin trouxe à luz a Teoria da Evolução (On the origin of species, Darwin, 1859). Curiosamente, o naturalista foi um dos primeiros pesquisadores a explorar e 
divulgar massivamente a fotografia em um tratado científico, ao descrever e analisar as expressões faciais através de imagens (Darwin, 1872).

Um segundo marco importante para a fotografia científica ocorreu com o surgimento da era digital, particularmente a partir da década de 1990. A fotografia digital tornou a prática fotográfica menos custosa e mais acessível aos pesquisadores, refletindo em um crescimento exponencial na utilização fotografia como método científico em estudos biológicos. O rápido desenvolvimento tecnológico aumentou significantemente a qualidade e a diversidade de equipamentos fotográficos. Paralelamente, softwares de edição cada vez mais sofisticados possibilitaram a transformação de pixels e seus atributos (e.g. cor, luminosidade, contraste) em dados quantificáveis. Desta forma, não é de se estranhar que dentre os estudos fotográfico-biológicos existentes na literatura, aproximadamente $80 \%$ foram publicados ao longo dos últimos 30 anos.

\section{USO DA FOTOGRAFIA NA BIOLOGIA}

Atualmente a literatura científica conta com mais de duas centenas de estudos que utilizaram a fotografia como instrumento de pesquisa na biologia. Dentre as vantagens da fotografia científica biológica (FCB), destacam-se (1) a facilidade e eficiência na geração de dados, (2) o uso de metodologias não invasivas, (3) a capacidade de nos revelar características do mundo natural que são imperceptíveis ao sistema visual humano, (4) a possibilidade de investigar ambientes de difícil acesso e (5) estudar organismos raramente observados na natureza. A versatilidade da FCB tem sido explorada tanto para investigações específicas sobre determinados organismos, quanto em estudos de padrões gerais em nível de populações, comunidades e paisagens. A seguir veremos algumas técnicas e utilizações da FCB.

Macro e microfotografia: técnicas que utilizam objetivas macro, lupas e microscópios para gerar imagens com grandes ampliações (relação numérica entre $o$ tamanho real do assunto 
fotografado e o tamanho em que ele aparece no sensor da câmera), possibilitando a visualização de objetos extremamente pequenos. Bastante utilizadas em estudos sobre microrganismos e artrópodes, seres que constituem a maior diversidade de espécies viventes em nosso planeta e desempenham papel fundamental na estruturação e funcionamento dos ecossistemas. As fotografias podem ser individuais (obtidas através de uma única imagem) ou mais sofisticadas, através da composição de dezenas ou centenas de fotografias empilhadas e com diferentes áreas em foco (focus stacking, Brecko, 2014), gerando imagens com alta resolução de detalhes. A macro e a microfotografia são amplamente exploradas na taxonomia, como ilustração em descrições de espécies, bem como em estudos botânicos e zoológicos, na análise de pequenas estruturas e/ou comportamentos. McIlleron e Moor (2011), por exemplo, desenvolveram uma técnica fotográfica para auxiliar o estudo de insetos em voo, baseada na aquisição de fotografias de alta velocidade acoplada a sensores de movimento.
Fotografia remota: trata-se da fotografia automatizada através do uso de sensores e/ou controles remotos, possibilitando a investigação de locais de difícil acesso (e.g. ambiente aéreo, dossel de florestas e o fundo dos oceanos) e de animais raramente encontrados na natureza. Cutler e Swann (2006) escreveram uma revisão sobre o uso desta técnica em estudos ecológicos. A fotografia aérea, por exemplo, tem sido extensivamente utilizada para a caracterização e mapeamento de comunidades vegetais (e.g. You-Ching, 1980, Lucas et al., 2008; Carlyle et al., 2010, Chen et al., 2012). Esta técnica também foi utilizada para a detecção de danos causados por gafanhotos em plantações (Olfert et al., 1980) e para estimar o tamanho populacional de pinguins na Antártica (Fraser, 1999; Trathan, 2004). Estes e outros exemplos reforçam a importância da fotografia aérea como poderosa ferramenta em estudos de manejo ecológico (Morgan et al., 2010). A recente popularização de drones e o consequente barateamento da fotografia aérea provavelmente resultará em aumento substancial no uso da FCB em 
investigações científicas nos próximos anos. No ambiente terrestre, armadilhas fotográficas (camera trap) são amplamente utilizadas para a foto-identificação de mamíferos, auxiliando a desvendar a diversidade, ecologia populacional e os comportamentos destes animais. O oceano também já foi explorado através da fotografia remota. Muito do que se conhece sobre a diversidade e os comportamentos da fauna abissal, por exemplo, se deve ao uso de equipamentos fotográficos capazes de acessar o mar profundo e suportar pressões elevadas.

Fotografia subaquática: além de permitir o acesso à fauna abissal, a fotografia subaquática tem sido um importante instrumento metodológico na biologia marinha, seja para estudos comportamentais e ecológicos de cetáceos (Würsig e Jefferson 1990; Grellier et al., 2003), tubarões, arraias, (Marshall e Pierce 2012) e tartarugas marinhas, alguns destes em perigo de extinção (Schofield et al., 2008), seja para avaliar a densidade e diversidade de organismos planctônicos (Grosjean et al., 2004). A fotografia também possibilita a foto-identificação de indivíduos em espécies com alta variação morfológica intraespecífica, auxiliando no monitoramento de suas populações. Kitahara (2007) forneceu importantes discussões e perspectivas quanto ao uso da fotografia em pesquisas oceanográficas.

Fotografia time lapse: eventos que ocorrem ao longo do tempo podem ser revelados através do uso da fotografia time-lapse, que consiste em séries de imagens sequenciais captadas em intervalos de tempo pré-definidos. Esta técnica foi utilizada, por exemplo, para descrever períodos de atividade de aves (Weller e Derksen, 1972), variações sazonais no crescimento de corais (Barnes e Crossland, 1980), comportamento alimentar de anfípodes e echiúros (Hargrave, 1985; Bett e Rice, 1993), diversidade e frequência de visitantes florais (Suetsugu e Hayamizu, 2014; Nakase e Suetsugu, 2015), bem como para descrever padrões fenológicos de populações em ecossistemas florestais (Sonnentag et al., 2012). A fotografia time lapse também pode ser utilizada em 
estudos sobre o desenvolvimento de organismos, bem como contribuir para a ecologia da conservação ao possibilitar a análise do impacto humano em ambientes naturais ao longo do tempo.

Fotografia hemisférica: trata-se do uso de câmeras acopladas a objetivas grande angulares ou fisheye, que geram imagens com grandes ângulos de visão (até $180^{\circ}$ ). Amplamente utilizada em estudos sobre o dossel de florestas, auxiliando na aquisição de variáveis biofísicas, como a estrutura do dossel, cálculo de índices de área foliar, ou ainda para estimar a luminosidade em ambientes florestais. Estes dados são gerados através do uso de softwares específicos que calculam a frequência relativa de áreas escuras (vegetação) e claras (céu) nas fotografias obtidas. Quando utilizadas em conjunto, a fotografia hemisférica e a time-lapse podem elucidar mudanças e possíveis impactos em florestas ao longo do tempo. Glatthorn e Beckschäfer (2014) publicaram um protocolo para padronizar o uso de fotografias hemisféricas.
Fotografia ultravioleta (UV) e infravermelha (IR): o uso de filtros específicos e a adaptação de sensores de câmeras fotográficas nos possibilita a aquisição de imagens que captam comprimentos de onda UV e IR. Muitos animais, como a maioria dos insetos, aves, répteis e anfíbios, são capazes de enxergar o espectro UV. Desta forma, a fotografia UV torna possível a compreensão de como determinados organismos são percebidos levando-se em consideração o sistema visual de seus co-específicos, presas e/ou predadores. Esta ferramenta tem sido bastante explorada em estudos sobre camuflagem (Troscianko et al., 2017), variações de coloração entre indivíduos (McGaugh, 2008; Stevens et al., 2009; Luttrell et al., 2014), bem como na taxonomia (Ferris, 1972; Knüttel e Fielder 2000). Recentemente, Troscianko e Stevens (2015) desenvolveram um software gratuito para a análise de fotografias UV e obtenção de medidas de reflectância, coloração e padrões na natureza. A fotografia IR, por sua vez, tem sido utilizada como ferramenta de mapeamento e monitoramento em estudos envolvendo 
vegetação, solos e corpos d'água (Aber et al., 2009), bem como para detectar doenças causadas por vírus em plantas (Bawden, 1993).

Além das técnicas vistas acima, a FCB também pode ser utilizada em estudos específicos, como a caracterização da arquitetura de teias de aranhas (Langer e Eberhard, 1969; Eberhard, 1976; Burgess, 1977; Carico, 1977), documentação de espécies de artrópodes em inclusões de âmbar (Baker et al., 2003), técnica auxiliar para ilustrações botânicas (Simpson e Barnes, 2008), ou para estudos biométricos, permitindo a aquisição de medidas de área foliar (Rico-García et al., 2009) ou do tamanho de ovos (Bridge et al., 2007) e de grandes mamíferos (Wijeyamohan et al., 2012). O objetivo deste tópico foi apresentar uma amostra das possibilidades de uso da FCB na biologia, sem a pretensão de esgotar o assunto ou realizar uma revisão sobre o tema. Muitos estudos novos são publicados a cada ano e certamente novos trabalhos estarão presentes na literatura quando você estiver lendo este artigo.

\section{OLHAR CRÍTICO}

Apesar de ser uma prática científica relativamente recente, o uso da FCB tem crescido significativamente ao longo das últimas décadas. Como qualquer atividade em expansão, torna-se imprescindível a avaliação crítica quanto à confiabilidade dos dados obtidos e sua aceitação pela comunidade científica. Augusto et al. (2013), por exemplo, demonstraram que não é possível usar a fotografia para determinar o sexo de baleias-piloto, devido à similaridade morfológica das barbatanas entre machos e fêmeas. Ksiksi e ElKeblawy (2013) provaram que a fotografia digital não é eficaz como metodologia alternativa à coleta em campo de vegetais em ecossistemas desérticos. Entretanto, o exemplo mais emblemático sobre como devemos ser cuidadosos ao utilizar a FCB é a atual discussão calorosa relacionada ao uso da fotografia como alternativa à coleta de espécimes em estudos taxonômicos, que pode ser acompanhada através de uma série de artigos recentemente publicados (veja Ceríaco, 2016; Pape, 2016; Aguiar et al., 2017; Faúndez, 2017; Garraffoni e Freitas, 2017; Grandcolas, 2017; Gutiérrez 
e Pine, 2017; Garrouste, 2017; Thorpe, 2017).

$\mathrm{O}$ ato fotográfico também pode resultar em impactos nos organismos estudados. Huang et al. (2011), por exemplo, demonstraram que sons produzidos pelas câmeras na hora do disparo afetam o comportamento do lagarto Anolis cristatellus (Dactyloidae). Os autores ressaltaram a importância de tomarmos cuidados e restrições quando fotografamos, para reduzir este tipo de interferência. Em contrapartida, Harasti e Gladstone (2013) verificaram que o uso do flash não modifica o comportamento do cavalo-marinho Hippocampus whitei (Syngnathidae). Estudos como estes são extremamente importantes e necessários para a criação de técnicas fotográficas não invasivas.

Tão importante quanto a aquisição de boas imagens em campo ou em laboratório é a adoção de fluxos de imagens adequados para garantir alta qualidade e confiabilidade das pesquisas. As etapas envolvidas no pósprocessamento das imagens são fundamentais para a organização dos dados coletados (edição), adequação das fotografias ao objetivo do estudo e às boas condutas éticas (tratamento) e segurança das fotografias contra falhas mecânicas e/ou eletrônicas (armazenamento das imagens). Selden (2014), por exemplo, sugeriu um modelo de fluxo de imagens para a fotografia de espécies fósseis. Rossner e Yamada (2004), por sua vez, escreveram uma excelente crítica sobre a ética na fotografia científica. Os autores trouxeram à tona exemplos de má conduta científica através do abuso na manipulação de imagens, ressaltando que este tipo de comportamento gera resultados não confiáveis.

Os exemplos supracitados reforçam a importância de avaliarmos a FCB desde a sua concepção ética e rigor metodológico, até a forma com que os resultados são apresentados. Isto abre um amplo leque de possibilidades para estudos futuros que auxiliem a consolidar a FCB como linha de pesquisa na biologia. Espera-se que sejam criados protocolos específicos para diferentes técnicas e organismos de estudo, com o objetivo de padronizar metodologias fotográfico-científicas e gerar dados 
confiáveis e comparáveis entre si. Além de atender às questões éticas e à boa qualidade científica, os novos métodos fotográficos devem ser fáceis de serem reproduzidos, apresentarem o menor custos de execução possível e levarem em consideração a variação das fotografias produzidas por diferentes marcas e modelos de equipamentos fotográficos.

\section{PERSPECTIVAS}

A fotografia é interdisciplinar e isto a permite transitar entre diversas áreas do conhecimento. Além desta versatilidade, possui alta capacidade adaptativa a temáticas e objetivos específicos. Dentre as perspectivas para o uso da FCB, gostaria de destacar alguns dos nichos da biologia ainda pouco explorados e/ou que se apresentam como campos férteis para a realização de investigações futuras mais aprofundadas:

(1) Educação, como ferramenta didática em sala de aula ou em trabalhos de educação ambiental (veja Borges et al., 2010; Sonohata e Sabino, 2015).
(2) Divulgação científica, auxiliando na disseminação do conhecimento de maneira clara e objetiva através de meios impressos e/ou eletrônicos (veja Sabino, 2009).

(3) Estudos de história natural, retratando eventos raros que eventualmente gerem insights para a formulação de hipóteses testáveis $a$ posteriori.

(4) Redes sociais e banco de imagens, estreitando os laços entre biólogos e cidadãos não cientistas (exemplo em Winterton et al., 2012) e auxiliando na compreensão de padrões globais (veja Leighton et al., 2016).

(5) Biomimética, através do estudo de sistemas visuais não humanos que possam servir como fonte de inspiração para o desenvolvimento de novas tecnologias e invenção de câmeras fotográficas mais sofisticadas (veja Caro et al., 2017).

(6) Metodologias não invasivas, seja em substituição a métodos tradicionais ou como ferramenta complementar em estudos ecológicos, botânicos, zoológicos, micológicos, comportamentais ou qualquer 
outra área relacionada à temática ambiental.

Em síntese, a invenção da fotografia revelou novos caminhos e horizontes para a Ciência. Apesar de atrair cada vez mais a atenção de biólogos como instrumento em pesquisas, o potencial metodológico da FCB ainda é pouco explorado, subutilizado e está fragmentado na literatura, dificultando as conexões entre os estudos. Este artigo representa um breve ponto de vista sobre a fotografia científica ambiental. Além disso, serve como um chamado para jovens biólogos e pesquisadores, com o objetivo de aumentar o interesse pelo assunto e estreitar o intercâmbio entre duas áreas do conhecimento altamente complementares e sinérgicas. Espero que em um futuro próximo sejam criados canais de discussão, reuniões científicas e cursos especializados visando a capacitação de pesquisadores neste fantástico mundo da FCB. Biólogos, peguem suas câmeras e bom trabalho!

\section{REFERÊNCIAS}

Aber JS, Aber SW, Buster L, Jensen WE, Sleezer RL. 2009. Challenge of infrared aerial photography: a digital update. Transactions of the Kansas Academy of Science. 112(1/2): 31-39.

Aguiar JJM, Santos JC, Urso-Guimarães MV. 2017. On the use of photography in Science and taxonomy: how images can provide a basis for their own authentication. Bionomina. 12: 44-47.

Augusto JF, Frasier TR, Whitehead H. 2013. Using photography to determine the sex in pilot whales (Globicephala melas) is not possible: Male and females have similar dorsal fins. Marine Mammal Science. 29(1): 213-220.

Baker RA, Chmielewski W, Evennett PJ. 2003. Amber inclusions of arthropods (particularly insects and mites) in European museums documentation and photography. Acta Zoologica Cracoviensia. 46: 399-405.

Barnes DJ, Crossland CJ. 1980. Diurnal and seasonal variation in the growth of a staghorn coral measured by time-lapse photography. Limnology and Oceanography. 25(6): 11131117

Bawden FC. 1993. Infra-red photography and plant virus diseases. Nature. 132: 168

Bett BJ, Rice AL. 1993. The feeding behavior of an abyssal echiuran revealed by in situ time-lapse photography. Deep Sea Research. 40(9): 17671779 .

Borges MD, Aranha JM, Sabino J. 2010. Nature photography as an instrument in Environmental Education. Ciência e Educação. 16(1): 149-161.

Bridge ES, Boughton RK, Aldredge PA, Harrison TJE, Bowman R, Schoech SJ. 2007. Measuring egg size using digital photography: testing 
Hoyt's method using Florida Scrub-Jay eggs. Journal of Field Ornithology. 78(1): 109-116.

Burgess JW. Orb-web photography outdoors: a graphic data-collection methodology. The American Midland Naturalist. 98(2): 499-503.

Carico JE. 1977. A simple dusting device for coating orb webs for field photography. Bulletin of British Arachnological Society. 4(2): 100.

Carlyle CN, Fraser LH, Haddow CM, Bings BA, Harrower W. 2010. The use of digital photos to access visual cover for wildlife in rangelands. Journal of Environmental Management. 91: 1366-1370.

Caro T, Stoddard MC, Stuart-Fox D. 2017. Animal coloration research: why it matters. Philosophical Transactions of the Royal Society B. 372: 20160333.

Ceríaco LMP, Gutiérrez EE, Dubois A. 2016. Photography-based taxonomy is inadequate, unnecessary, and potentially harmful for biological sciences. Zootaxa. 4196(3): 435-445.

Chen Q, Laurin GV, Battles JJ, Saah D. 2012. Integration of airbone lidar and vegetation types derived from aerial photography for mapping aboveground live biomass. Remote Sensing and Environment. 121: 108-117.

Cutler TL, Swann DE. 1999. Using remote photography in wildlife ecology: a review. Wildlife Society Bulletin. 27(3): 571-581.

Darwin C. 1859. On the origin of species. London (UK): John Murray.

Darwin C. 1982. The Expression of the Emotions in Man and Animals. London (UK): Harper Collins.

Eberhard WG. 1976. Photography of orb webs in the field. Bulletin of British Arachnological Society. 3(7): 200-204.
Faúndez EI. 2007. Photography-based taxonomy: Is it necessary to reform the Code, and what that exactly means? Zootaxa. 4247(3): 332-332.

Ferris CD. 1972. Ultraviolet photography as an adjunct to taxonomy. Journal of the Lepidopterists's Society. 26(4): 210-215.

Fraser WR, Carlson JC, Duley PA, Holm EJ, Patterson DL. 1999. Using kite-based aerial photography for conducting Adélie penguin censuses in Antarctica. Waterbirds. 22(3): 435440.

Garraffoni ARS, Freitas AVL. 2017. Photos belong in the taxonomic Code. Science. 355: 805.

Garrouste R. 2017. The "wild shot": photography for more biology in natural history collections, not for replacing vouchers. Zootaxa. 4269: 453454.

Glatthorn J, Beckschäfer P. 2014. Standardizing the protocol for hemispherical photographs: accuracy assessment of binarization algorithms. PLoS ONE. 9(11): e111924.

Grandcolas P. 2017. Loosing the connection between the observation and the specimen: a byproduct of the digital era or a trend from general biology? Bionomina. 12: 57-62.

Grellier K, Hammond PS, Wilson B, Sanders-Reed CA, Thompson PM. 2003. Use of photoidentification data to quantify mother-calf association patterns in bottlenose dolphins. Canadian Journal of Zoology. 81: 1421-1427.

Grosjean P, Pischeral M, Warembourg C, Gorsky G. 2004. Enumeration, measurement,

and identification of net zooplankton samples using the zooscan digital imaging system. ICES Journal of Marine Science. 61: 518-525.

Gutiérrerz EE, Pine RH. 20107. Specimen collection crucial to taxonomy. Science. 355: 1275 
Hargrave BT. 1985. Feeding rates of abyssal scavenging amphipods (Eurythenes gryllus) determined in situ by time-lapse photography. Deep Sea Research. 32(4): 443-450.

Harasti D, Gladstone W. 2013. Does underwater flash photography affect the behaviour, movement and site persistance of seahorses? Journal of Fish Biology. 83: 1344-1353.

Huang B, Lubarsky K, Teng T, Brumstein DT. 2011. Take only pictures, leave only...fear? The effects of photography on the West Indian anole Anolis cristatellus. Current Zoology. 57(1): 77-82.

Kitahara EM. 2007. O uso da fotografia e da imagem digital em pesquisas oceanográficas: novos rumos proporcionados pela evolução do processo digital. Conexão - Comunicação e Cultura. 6(12): 125-137.

Knüttel H, Fielder K. 2000. On the use of ultraviolet photography and ultraviolet wing patters in butterfly morphology and taxonomy. Journal of the Lepidopterist's Society. 54(4): 137-144.

Ksiksi TS, El-Keblawy AA. 2013. Floral diversity in desert ecosystems: Comparing field sampling to image analyses in assessing species cover. BMC Ecology. 13: 22.

Langer RM, Eberhard WG. Laboratory photography of spider silk. American Zoologist. 9(1): 97-101.

Leighton GRM, Hugo PS, Roulin A, Amar A. 2016. Just Google it: assessing the use of Google Images to describe geographical variation in visible traits of organisms. Methods in Ecology and Evolution. 7(9): 1060-1070.

Lucas R, Bunting P, Paterson M, Chisholm L. 2008. Classification of Australian forest communities using aerial photography, CASI and HyMap data. Remote Sensing of Environment. 112: 2088-2103.
Luttrell SAM, Gonzalez ST, Lohr B, Greenberg R. 2014. Digital photography quantifies plumage variation and salt marsh melanism among Song Sparrow (Melospiza melodia) subspecies of the San Francisco Bay. The Auk. 132: 277-287.

Marshall AD, Pierce SJ. 2012. The use of photographic identification in sharks and rays. Journal of Fish Biology. 80: 1361-1379.

McGaugh SE. 2008. Color variation among habitat types in the Spiny Softshell Turtles (Trionychidae: Apalone) of Cuatrociénegas, Coahuila, México. Journal of Herpetology. 42(2): 347-353.

McIlleron WG, Moor FC. 2011. Photography of Trichoptera in flight. Zoosymposia. 5: 297-318.

Nakase Y, Suetsugu K. 2015. Technique to detect flower-visiting insects in video monitoring and time-lapse photography data. Plant Species Biology. 31(2): 148-152.

Olfert OO, Gage SH, Mukerji MK. 1980. Aerial photography for detection and assessment of grasshopper (Orthoptera: Acrididae) damage to small grain crops in Saskatchewan. The Canadian Entomologist. 112(6): 559-566.

Pape T. 2016. Taxonomy: species can be named from photos. Nature. 537: 307.

Prodger P. 2009. Darwin's camera: Art and photography in the theory of evolution. New York (USA): Oxford University Press. 320 p.

Rico-García E, Hernández-Hernández F, SotoZarazúa GM, Herrera-Ruiz G. 2009. Two new methods for the estimation of leaf area using digital photography. International Journal of Agriculture and Biology. 11: 397-400.

Rossner M, Tamada KM. 2004. What's in a picture? The temptation of image manipulation. The Journal of Cell Biology. 166(1): 11-15. 
Sabino J. 2009. Técnica e ética da fotografia do comportamento animal: dos pioneiros à era digital. Oecologia Brasiliensis. 13: 209-221.

Schofield G, Katselidis KA, Dimopoulos P, Pantis JD. 2008. Investigating the viability of photoidentification as an objective tool to study endangered sea turtle populations. Journal of Experimental Marine Biology and Ecology. 360: 103-108.

Selden PA. 2014. A workflow for digital photography of fossil specimens. The Geological Curator. 10(2): 93-98.

Simpson N, Barnes PG. 2008. Photography and contemporary botanical illustration. Botanical Magazine. 25(3): 258-280.

Sonnentag O, Hufkens K, Teshera-Sterne C, Young $\mathrm{AM}$, Friedl $\mathrm{M}$, Braswell $\mathrm{BH}$, Milliman $\mathrm{T}$, O’Keefe J, Richardson AD. 2012. Digital repeat photography for phenological research in forest ecosystems. Agricultural and Forest Meteorology. 152: 159-177.

Sonohata RI, Sabino J. 2015. Use of photography in the understanding of biodiversity elements of the Pantanal and Bonito region. UNOPAR Científica Ciências Humanas e Educação. 16: 437-444.

Stevens M, Stoddard MC, Highan JP. 2009. Studying primate color: towards visual systemdependent methods. International Journal of Primatology. 30: 893-917.

Suetsugu K, Hayamizu M. 2014. Moth floral visitors of the three rewarding Platanthera orchids revealed by interval photography with a digital camera. Journal of Natural History. 48(17-18): 1103-1109.

Talbot WHF. 1844. The Pencil of Nature. London (UK): Longman, Brown, Green \& Longmans.
Thorpe SE. 2017. Is photography-based taxonomy really inadequate, unnecessary, and potentially harmful for biological sciences? A reply to Ceríaco et al. (2016). Zootaxa. 4226 (3): 449450 .

Troscianko J, Stevens M. 2015. Image calibration and analysis toolbox - a free software suite for objectively measuring reflectance, colour and pattern. Methods in Ecology and Evolution. 6: 1320-1331.

Troscianko J, Skelhorn J, Stevens M. 2017. Quantifying camouflage: how to predict detectability from appearance. BMC Evolutionary Biology. 17:7.

Trathan PN. 2004. Image analysis of color aerial photography to estimate penguin population size. Wildlife Society Bulletin. 32(2): 332-343.

Weller MW, Derksen DV. 1972. Use of time-lapse photography to study nesting activities of birds. The Auk. 89(1): 196-200.

Winterton SL, Guek HP, Brooks SJ. 2012. A charismatic new species of green lacewing discovered in Malaysia (Neuroptera, Chrysopidae): the confluence of citizen scientist, online image database and cybertaxonomy. ZooKeys. 214: 1-11.

Wijeyamohan S, Sivakumar V, Read B, Schimitt D, Krishnakumar S, Santlapillai C. 2012. A simple technique to estimate linear body measurements of elephants. Current Science. 102(1): 26-28.

Würsig B, Jefferson TA. 1990. Methods of photoidentification for small cetaceans. Report of the International Whaling Commission. 12: 43-52.

You-Ching F. 1980. Aerial photo and landsat image use in forest inventory in China. Photogrammetric Engineering and Remote Sensing. 46(11): 1421-1424. 